\title{
Fast 2.5D Finite Element Simulations of Borehole Resistivity Measurements
}

\author{
Ángel Rodríguez-Rozas · David Pardo · Carlos Torres-Verdín
}

Received: May 1, 2018/ Accepted: date

\begin{abstract}
We develop a rapid 2.5-dimensional (2.5D) finite element method for simulation of borehole resistivity measurements in transversely isotropic (TI) media. The method combines arbitrary high-order $H^{1}$ and $\mathbf{H}$ (curl)-conforming spatial discretizations. It solves problems where material properties remain constant along one spatial direction, over which we consider a Fourier series expansion and each Fourier mode is solved independently.

We propose a novel a priori method to construct quasi-optimal discretizations in physical and Fourier space. This construction is based on examining the analytical (fundamental) solution of the 2.5D formulation over multiple homogeneous spaces and assuming that some of its properties still hold for the 2.5D problem over a spatially heterogeneous formation. In addition, a simple parallelization scheme over multiple measurement positions provides efficient scalability.

Our method yields accurate borehole logging simulations for realistic synthetic examples, delivering simulations of borehole resistivity measurements at a rate faster than 0.05 seconds per measurement location along the well trajectory on a 96-core computer.
\end{abstract}

Ángel Rodríguez-Rozas*

BCAM - Basque Center for Applied Mathematics, Bilbao, Spain

E-mail: angel.rodriguez.rozas@gmail.com;

*Corresponding author

ORCiD: 0000-0002-9703-1043

David Pardo

University of the Basque Country (UPV/EHU), Leioa, Spain BCAM - Basque Center for Applied Mathematics, Bilbao, Spain

IKERBASQUE (Basque Foundation for Sciences), Bilbao, Spain

Carlos Torres-Verdín

The University of Texas at Austin, Austin, Texas, USA
Keywords Borehole resistivity measurements · fast 2.5D simulations · finite element method · logging while drilling

Mathematics Subject Classification (2000) 65Y05. $65 \mathrm{Y} 99 \cdot 65 \mathrm{~T} 99 \cdot 86 \mathrm{~A} 04$

\section{Introduction}

Borehole geophysical measurements, also known as well logs, often comprise hundreds or even thousands of measurements acquired at different locations along the well trajectory $[14,5,16,19]$. Thus, their computer simulation involves the solution of multiple three-dimensional (3D) problems, which is computationally expensive. This prevents their real-time inversion $[32,4,12]$, needed for well geosteering applications $[17,5]$.

In order to reduce the computational cost, it is possible to decrease the problem dimensionality by means of a Fourier or a Hankel series expansion along one or two spatial dimensions, leading to the so-called $2.5 \mathrm{D}$ $[33,30,22,25,31,28,24,1]$ and $1.5 \mathrm{D}[4,27]$ formulations, respectively. $1.5 \mathrm{D}$ problems can be rapidly solved semianalytically [18]; however, they are inaccurate when dealing with geological faults and/or other high-dimensional spatial features that may appear in rock formations [4].

In this work, we focus on $2.5 \mathrm{D}$ formulations, which seem to provide an adequate balance between model accuracy and speed of simulations for a large number of borehole geophysical conditions.

Efficient 2.5D simulations for real-time well geosteering inversion require spatial discretizations (grids) that are often challenging to design. One possibility is the use of a posteriori goal-oriented adaptive methods [10, $6,21]$. Unfortunately, these adaptive processes require a 
significant amount of CPU time, preventing them from being practical for real-time inversion. On the other hand, uniform grids and/or traditional mesh generators often deliver unnecessarily large systems that are costly to solve. Even the use of iterative solvers (e.g., [23]) is insufficient to mitigate such elevated computational cost.

In this paper, we propose a method to design a priori quasi-optimal 2.5D spatial discretizations that provide accurate simulations in a small fraction of a CPU second per logging position. The main idea is based on analyzing a sequence of (fundamental) solutions over a homogeneous space for the 2.5D formulation, for which it is possible to construct optimal discretizations and employ them to design automatically on the fly quasioptimal grids for spatially heterogeneous problems and transversely isotropic (TI) media. Because the analytical Fourier transform of the 3D solution over homogeneous media [20] is unavailable and elusive, we derive it analytically directly from the $2.5 \mathrm{D}$ formulation of Maxwell's equations in homogeneous media (see the Appendix). Some key features of our method include: (a) we quasi-optimally determine the domain and element sizes for each Fourier mode by analizing a priori the fundamental solution over a homogeneous space; (b) we establish a criterion to assign different polynomial orders of approximation to each element; (c) we interpolate the solution at measurement locations with some Fourier modes instead of computing them all via a FEM; (d) we quasi-optimally subdivide the well trajectory into several sub-trajectories, employing a single computational mesh for each sub-trajectory; and (e) we introduce a trivial parallelization based on dynamic scheduling of independent tasks. The proposed method is simple to implement and supports arbitrary highorder $H^{1}$ - and $\mathbf{H}$ (curl)-conforming mixed discretizations.

The rest of this article is organized as follows: We first describe the 3D logging problem in detail. Next, we introduce the 2.5D Fourier Finite Element formulation. Next, we introduce the main features of our 2.5D discretization method. Subsequently, we describe numerical results obtained on a parallel computer and compare their accuracy to those corresponding to a $1.5 \mathrm{D}$ semianalytical method. Finally, we summarize our conclusions and future work. In the Appendix, we describe the fundamental solution for the 2.5D formulation over a homogeneous space.

\section{Problem description}

We consider the LWD instrument shown in Fig. 1 [15]. Transmitters and receivers are symmetrically arranged about the tool center. For each transmitter and receiver pair, triaxial logging instruments generate measurements comprising all possible combinations of transmitter and receiver orientations [11]. Namely, XX, XY, $\mathrm{XZ}, \mathrm{YX}, \mathrm{YY}, \mathrm{YZ}, \mathrm{ZX}, \mathrm{ZY}$, and ZZ, where the first index designates the transmitter orientation and the second one the receiver orientation. We refer to $\mathbf{H}^{(Y Z)}$ as the YZ magnetic field component.

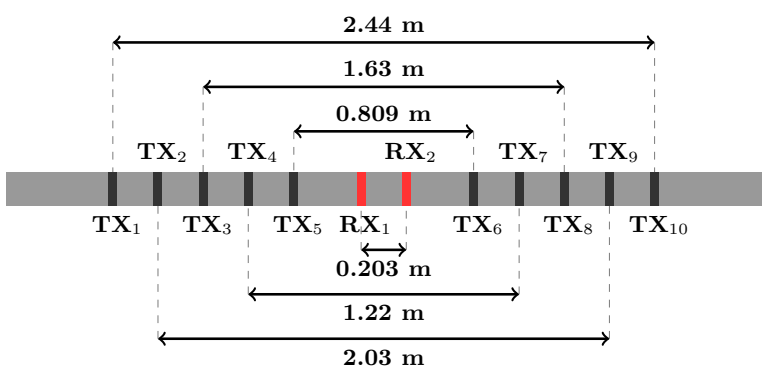

Fig. 1: LWD instrument composed of two receivers and ten transmitters symmetrically arranged from the center of the tool $\mathbf{x}_{\mathrm{t}}$. Each transmitter and receiver has three mutually orthogonal antenna coils [17].

In all our numerical examples, we examine receivers $\mathbf{R X}_{1}$ and $\mathbf{R X}_{2}$ and transmitters $\mathbf{T X}_{5}$ and $\mathbf{T} \mathbf{X}_{6}$. This combination provides the shallowest depth of investigation.

$$
\text { Vector } \mathbf{X}_{\text {pos }}:=\left\{\mathbf{x}_{\mathrm{t}}^{(i)}\right\}_{i=1, \ldots, N_{\text {pos }}} \text { defines the logging }
$$
trajectory composed of $N_{\text {pos }}$ tool positions.

We consider two frequencies of operation: $2 \mathrm{MHz}$ and $400 \mathrm{KHz}$. The induced magnetic field $\mathbf{H}$ measured at the two receivers is used to compute the attenuation and phase difference (or shift). The signal is simmetrized by averaging the measurements originating from each transmitter pair symmetrically arranged with respect to $\mathbf{x}_{\mathrm{t}}$. The resulting quantities are often converted into apparent resistivities using a simple look-up table algorithm based on the tool response in a homogeneous isotropic medium, which is analytically known [3].

We define $\mathbf{H}_{Y Z}^{m, n}\left(x_{\mathrm{t}}^{(i)}\right)$ as the YZ magnetic field component induced by transmitter $\mathbf{T} \mathbf{X}_{m}$ measured at at $\mathbf{R X}_{n}$, when the logging instrument is located at $\mathbf{x}_{\mathbf{t}}^{(i)}$. The attenuation and phase differences for the co-axial component $\left(\mathbf{H}_{Z Z}\right)$ are obtained by taking the real and imaginary parts, respectively, of the following (nonlinear) quantity of interest (QoI)

$q_{Z Z}\left(\mathbf{x}_{\mathrm{t}}^{(i)}\right)=\frac{1}{2}\left(\log \frac{\mathbf{H}_{Z Z}^{5,1}\left(\mathbf{x}_{\mathrm{t}}^{(i)}\right)}{\mathbf{H}_{Z Z}^{5,2}\left(\mathbf{x}_{\mathrm{t}}^{(i)}\right)}+\log \frac{\mathbf{H}_{Z Z}^{6,1}\left(\mathbf{x}_{\mathrm{t}}^{(i)}\right)}{\mathbf{H}_{Z Z}^{6,2}\left(\mathbf{x}_{\mathrm{t}}^{(i)}\right)}\right)$. 


\section{The 2.5D Fourier FE method}

We consider the following reduced wave equation in terms of the magnetic field $\mathbf{H}$ with an assumed timeharmonic dependence $e^{j \omega t}$,

$$
\nabla \times\left(\hat{\boldsymbol{\sigma}}^{-1} \nabla \times \mathbf{H}\right)+j \omega \boldsymbol{\mu} \mathbf{H}=-j \omega \boldsymbol{\mu} \mathbf{M}^{\mathrm{imp}},
$$

defined over an unbounded domain $\Omega$ with a transversely isotropic (TI) medium, where $j$ is the imaginary unit, $\boldsymbol{\mu}=\mu_{0} \mathbf{I}$ is the free-space magnetic permeability tensor, $\mu_{0}=4 \pi 10^{-7} N / A^{2}, \mathbf{I}$ is the $3 \times 3$ identity matrix, $\hat{\boldsymbol{\sigma}}:=(\boldsymbol{\sigma}+j \omega \varepsilon), \boldsymbol{\sigma}$ is the piecewise-constant conductivity diagonal tensor of the medium of the form

$$
\boldsymbol{\sigma}_{f}=\left(\begin{array}{ccc}
\sigma_{h} & 0 & 0 \\
0 & \sigma_{h} & 0 \\
0 & 0 & \sigma_{v}
\end{array}\right)
$$

$\boldsymbol{\rho}=\boldsymbol{\sigma}^{-1}$ is the resistivity tensor, $\boldsymbol{\varepsilon}=\varepsilon_{0} \mathbf{I}, \varepsilon_{0} \approx$ $8.85 \times 10^{-12} \mathrm{~F} / \mathrm{m}$ is the constant permittivity of the medium, $\omega=2 \pi f$ is the angular frequency, and $f$ is the frequency of operation of the resistivity instrument. Finally, $\mathbf{M}^{\text {imp }}$ is the time-harmonic directed magnetic dipole source excited by a tri-axial transmitter coil mounted on the logging instrument and located at $\left(x_{0}, y_{0}, z_{0}\right)$, mathematically defined as $\mathbf{M}^{\mathbf{i m p}}=\delta(x-$ $\left.x_{0}\right) \delta\left(y-y_{0}\right) \delta\left(z-z_{0}\right)\left[M_{x}, M_{y}, M_{z}\right]^{T}$, where $M_{x}, M_{y}$, and $M_{z}$ are positive real constants. We truncate the open domain $\Omega$ far away from the source by introducing an artificial boundary $\Gamma$ for which it suffices to impose a Perfect Magnetic Conductor (PMC) (see [9]) boundary condition:

$\mathbf{n} \times \mathbf{H}=\mathbf{0}$ on $\Gamma$.

In this work, we truncate our domain $\Omega$ using a tensor product box of size $L_{x} \times L_{y} \times L_{z}$ so that $\Omega=$ $\left[-L_{x} / 2, L_{x} / 2\right] \times\left[-L_{y} / 2, L_{y} / 2\right] \times\left[-L_{z} / 2, L_{z} / 2\right]$.

To introduce a variational formulation suitable for FE computations, we first define the continuous linear tangential trace mapping of a vector field $\mathbf{F}$ over $\Gamma$ as

$$
\gamma_{\Gamma}(\mathbf{F}):=\left.(\mathbf{n} \times \mathbf{F})\right|_{\Gamma}
$$

The corresponding tangential projection operator is denoted as:

$$
\pi_{\Gamma}(\mathbf{F}):=\mathbf{n} \times\left.(\mathbf{n} \times \mathbf{F})\right|_{\Gamma} .
$$

We define the following $\boldsymbol{H}(\mathbf{c u r l} ; \Omega)$-conforming functional spaces

$$
\begin{aligned}
& \boldsymbol{H}(\operatorname{curl} ; \Omega)=\left\{\mathbf{F}=\left(F_{x}, F_{y}, F_{z}\right) \in\left(L^{2}(\Omega)\right)^{3}\right. \\
&\left.: \nabla \times \mathbf{F} \in\left(L^{2}(\Omega)\right)^{3}\right\} . \\
& \boldsymbol{H}_{\Gamma}(\operatorname{curl} ; \Omega)=\left\{\mathbf{F} \in \boldsymbol{H}(\operatorname{curl} ; \Omega): \gamma_{\Gamma}(\mathbf{F})=\mathbf{0}\right\},
\end{aligned}
$$

where $\boldsymbol{H}(\mathbf{c u r l} ; \Omega)$ is a complex Hilbert space endowed with the inner product

$$
\begin{aligned}
& \left(\mathbf{F}_{\mathbf{1}}, \mathbf{F}_{\mathbf{2}}\right)_{\boldsymbol{H}(\operatorname{curl} ; \Omega)}:= \\
& \quad\left(\operatorname{curl}_{\mathbf{1}}, \operatorname{curl}_{\mathbf{2}}\right)_{\left(L^{2}(\Omega)\right)^{3}}+\left(\mathbf{F}_{\mathbf{1}}, \mathbf{F}_{\mathbf{2}}\right)_{\left(L^{2}(\Omega)\right)^{3}} \\
& \quad=\int_{\Omega}{\overline{\nabla \times \mathbf{F}_{\mathbf{1}}}}^{T} \cdot \nabla \times \mathbf{F}_{\mathbf{2}} d \Omega+\int_{\Omega} \overline{\mathbf{F}}^{T} \cdot \mathbf{F}_{\mathbf{2}} d \Omega,
\end{aligned}
$$

and the associated norm

$$
\|\mathbf{F}\|_{\boldsymbol{H}(\mathbf{c u r l} ; \Omega)}=\left(\|\mathbf{F}\|_{\left(L^{2}(\Omega)\right)^{3}}^{2}+\|\operatorname{curl} \mathbf{F}\|_{\left(L^{2}(\Omega)\right)^{3}}^{2}\right)^{1 / 2} .
$$

In the above, $\overline{\mathbf{F}}$ denotes the complex conjugate of $\mathbf{F}$. By pre-multiplying Eq. (2) with an arbitrary test function $\mathbf{F} \in \boldsymbol{H}_{\Gamma}(\mathbf{c u r l} ; \Omega)$, integrating over $\Omega$ and applying integration by parts, we obtain the following $3 \mathrm{D}$ variational formulation: Find $\mathbf{H} \in \boldsymbol{H}_{\Gamma}(\operatorname{curl} ; \Omega)$ such that:

$$
\begin{aligned}
& \left(\nabla \times \mathbf{F}, \hat{\boldsymbol{\sigma}}^{-1} \nabla \times \mathbf{H}\right)_{\left(L^{2}(\Omega)\right)^{3}}+j \omega \boldsymbol{\mu}(\mathbf{F}, \mathbf{H})_{\left(L^{2}(\Omega)\right)^{3}}= \\
& -j \omega \boldsymbol{\mu}\left(\mathbf{F}, \mathbf{M}^{\mathbf{i m p}}\right)_{\left(L^{2}(\Omega)\right)^{3}} \quad \forall \mathbf{F} \in \boldsymbol{H}_{\Gamma}(\mathbf{c u r l} ; \Omega) .
\end{aligned}
$$

Note that the boundary term in the above formulation vanishes due to the imposed boundary condition expressed in Eq. (3).

We now derive a $2.5 \mathrm{D}$ variational formulation. Assuming that material properties are homogeneous along one spatial direction, e.g., $y$-axis, we reduce the $3 \mathrm{D}$ variational formulation described in Eq. (8) to a $2.5 \mathrm{D}$ variational problem as follows: we first introduce notation to distinguish our tensor product domain given along the $y$-direction $\left(\Omega_{y}\right)$ from that along the $x$ - and $z$-directions $\left(\Omega_{x, z}\right)$, as $\Omega:=\Omega_{y} \times \Omega_{x, z}$, and the corresponding boundary decomposition $\Gamma:=\Gamma_{y} \times \Gamma_{x, z}$. We now represent the magnetic field solution as the following series expansion using complex exponentials (a.k.a. Fourier basis functions)

$\mathbf{H}(x, y, z):=\sum_{\beta=-\infty}^{+\infty} \mathbf{H}_{\beta}(x, z) e^{j 2 \pi \beta y / L_{y}}$,

Using Eq. (9), a mono-modal test function $\mathbf{F}(x, y, z):=$ $\frac{1}{L_{y}} \mathbf{F}_{\beta}(x, z) e^{j \beta y 2 \pi / L_{y}}$, and the well-known orthogonality relation for complex exponential functions on the interval $\left(-L_{y} / 2, L_{y} / 2\right)$ given by

$$
\frac{1}{L_{y}} \int_{-L_{y} / 2}^{L_{y} / 2} e^{-j \beta_{1} y 2 \pi / L_{y}} e^{j \beta_{2} y 2 \pi / L_{y}} d y=\delta_{\beta_{1} \beta_{2}},
$$

the variational formulation of Eq. (8) becomes: Find $\mathbf{H}(x, y, z)=\sum_{\beta=-\infty}^{+\infty} \mathbf{H}_{\beta}(x, z) e^{j \beta y 2 \pi / L_{y}}$ such that, for 
every $\beta \in(-\infty, \infty), \mathbf{H}_{\beta} \in \boldsymbol{H}_{\Gamma_{x, z}}\left(\operatorname{curl}^{\beta} ; \Omega_{x, z}\right)$ and:

$$
\begin{aligned}
& \left(\nabla^{\beta} \times \mathbf{F}_{\beta}, \hat{\boldsymbol{\sigma}}^{-1} \nabla^{\beta} \times \mathbf{H}_{\beta}\right)_{\left(L^{2}\left(\Omega_{x, z}\right)\right)^{3}} \\
& +j \omega \boldsymbol{\mu}\left(\mathbf{F}_{\beta}, \mathbf{H}_{\beta}\right)_{\left(L^{2}\left(\Omega_{x, z}\right)\right)^{3}}= \\
& -j \omega \boldsymbol{\mu}\left(\mathbf{F}_{\beta}, \mathbf{M}_{\beta}^{\mathbf{i m p}}\right)_{\left(L^{2}\left(\Omega_{x, z}\right)\right)^{3}} \\
& \quad \forall \mathbf{F}_{\beta} \in \boldsymbol{H}_{\Gamma_{x, z}}\left(\mathbf{c u r l}^{\beta} ; \Omega_{x, z}\right),
\end{aligned}
$$

where

$\mathbf{M}_{\beta}^{\mathrm{imp}}=\frac{1}{L_{y}} \delta\left(x-x_{0}\right) \delta\left(z-z_{0}\right)\left[M_{x}, M_{y}, M_{z}\right]^{T} e^{j 2 \pi \beta y_{0} / L_{y}}$,

$\nabla^{\beta} \times \mathbf{F}:=$

$$
\left(j \beta \frac{2 \pi}{L_{y}} F_{z}-\frac{\partial F_{y}}{\partial z}, \frac{\partial F_{x}}{\partial z}-\frac{\partial F_{z}}{\partial x}, \frac{\partial F_{y}}{\partial x}-j \beta \frac{2 \pi}{L_{y}} F_{x}\right),
$$

and the following functional spaces are used:

$$
\begin{aligned}
& \boldsymbol{H}\left(\mathbf{c u r l}^{\beta} ; \Omega_{x, z}\right):= \\
& \quad\left\{\mathbf{F}=\left(F_{x}, F_{y}, F_{z}\right) \in\left(L^{2}\left(\Omega_{x, z}\right)\right)^{3}: F_{y} \in H^{1}\left(\Omega_{x, z}\right)\right. \\
& \left.\quad \text { and } \nabla \times\left(F_{x}, F_{z}\right) \in\left(L^{2}\left(\Omega_{x, z}\right)\right)^{2}\right\}, \\
& \boldsymbol{H}_{\Gamma_{x, z}}\left(\operatorname{curl}^{\beta} ; \Omega_{x, z}\right):= \\
& \quad\left\{\mathbf{F} \in \boldsymbol{H}\left(\operatorname{curl}^{\beta} ; \Omega_{x, z}\right): \gamma_{\Gamma}(\mathbf{F})=\mathbf{0}\right\} .
\end{aligned}
$$

The selection of exponential functions along the $y$-direction for both test and trial functions, together with their orthogonality relations described in Eq. (10), allows us to decouple the original $3 \mathrm{D}$ problem into a set of independent 2D problems, one for each Fourier mode $\beta$. Additionally, this choice of basis functions allows for exponential convergence in terms of the number of Fourier modes, provided that the solution is sufficiently smooth.

\section{Method}

The proposed method generates a spatial discretization based on the analysis of multiple solutions over a homogeneous space with properties defined below. Once the method is discretized, we employ a direct solver (see [26]) to solve the subsequent linear system of equations via an LU factorization.

Domain truncation The domain size is selected to ensure that the solution at receivers is unaffected by boundary reflections. At the same time, we want this size to be as small as possible to save computational effort. To that end, we consider the worst-case scenario given by the slowest decay that can occur in our spatial domain. This corresponds to the fundamental solution $\mathbf{H}^{f}$ over a homogeneous space governed by a transversely isotropic
(TI) conductivity tensor with the smallest values on each direction from the range of all feasible rock electrical conductivities considered in our problem. Then, we compute the magnitude of the solution $\left|\mathbf{H}^{f}\right|$ at the farthest receiver with respect to the active transmitter. Finally, we select $L_{x}, L_{y}$ and $L_{z}$ so that at every point over the boundary, $\left|\mathbf{H}^{f}\right|$ is at least two orders of magnitude smaller than the solution $\left|\mathbf{H}^{f}\right|$ at the receiver located farthest away from the active transmitter.

In the $2.5 \mathrm{D}$ formulation, this condition depends both upon the conductivity tensor $\boldsymbol{\sigma}_{f}$ and the Fourier mode number $\beta$. From the convergence of the Fourier series, it is expected that the higher the Fourier mode the fastest the decay of $\left|\mathbf{H}_{Z Z}^{f}(\beta)\right|$ (see Fig.(2) for the real part). Thus, we truncate the series of Eq. (9) when the magnetic field at the receivers is sufficiently small in a homogeneous media (see [30]).

Consequently, $L_{x}$ and $L_{z}$ are functions of the Fourier mode $\beta: L_{x}(\beta)$ and $L_{z}(\beta)$. For the $y$-direction, the length of the Fourier domain $L_{y}$ is made constant for all Fourier modes: We select the maximum domain size within the range of Fourier modes considered when truncating the series in Eq. (9), $\beta \in\left[-N_{F}, \ldots, N_{F}\right], N_{F} \in$ $\mathbb{N}^{+}$, i.e., $L_{y}=\max _{\beta \in\left[-N_{F}, \ldots, N_{F}\right]} L_{y}(\beta)=S_{y}(0)$.

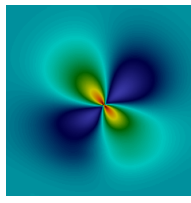

(a) $\beta=0$.

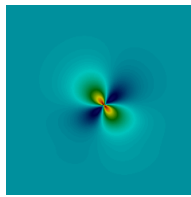

(e) $\beta=4$.

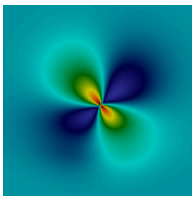

(b) $\beta=1$.

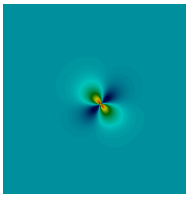

(f) $\beta=6$.

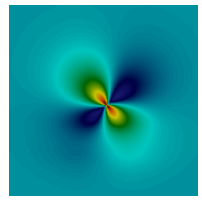

(c) $\beta=2$.

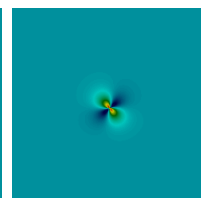

(g) $\beta=8$.

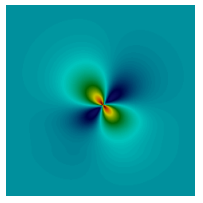

(d) $\beta=3$.

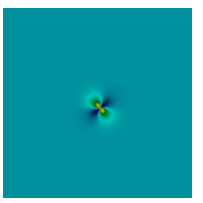

(h) $\beta=10$.
Fig. 2: Real-part of $\mathbf{H}_{Z Z}^{f}(\beta)$ for different Fourier modes $\beta$.

Computational mesh and element sizes We employ rectilinear non-uniform tensor-product meshes with variable element sizes (see Fig. 3). This enables the construction of simple quadrilateral elements using tensorproduct basis functions.

In order to minimize the numerical error at receivers, we bound the logging trajectory into a quadrilateral of lengths $l_{x}$ and $l_{z}$ (in $\mathrm{m}$ ) along the $x$ - and $z$-directions, respectively, including an extra space in every direction (in the figure, of the size of one extra element per side). 


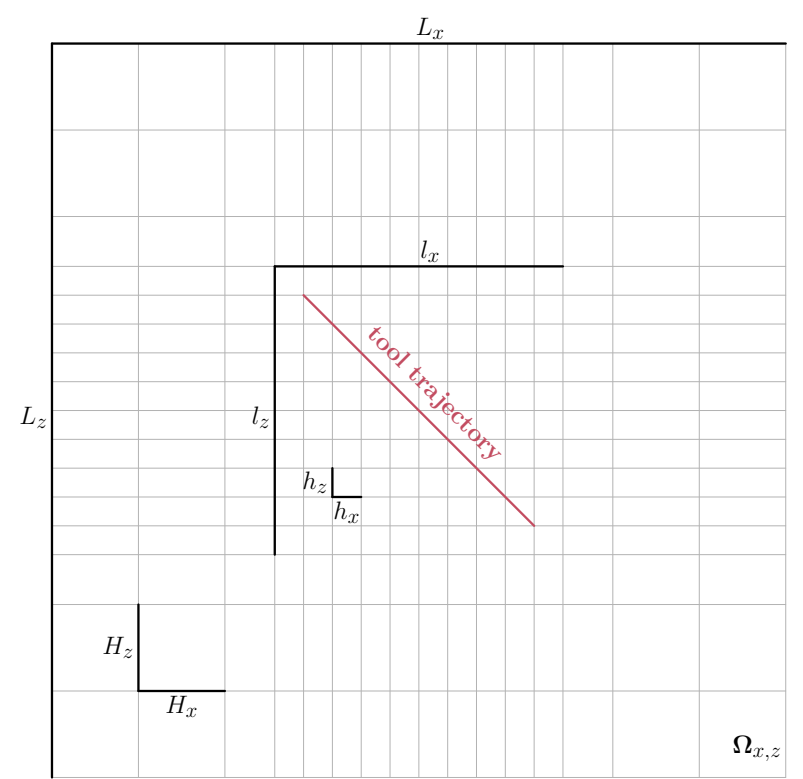

Fig. 3: Non-uniform tensor-product based mesh structure.

The elements contained therein feature the smallest element size, given by $h_{x} \times h_{z}$. Typically, $h_{x}=h_{z}=h$. To construct the elements in the rest of the domain, we implement a geometrical progression until reaching the largest element size $H_{x} \times H_{z}$, where typically $H_{x}=H_{z}=H=3 h$.

In order to select the element size $h$, we first define the set of electrical conductivities $\sigma_{\text {set }}=\sigma_{1}, \ldots, \sigma_{n}$ (or resistivities) present in our problem. For each conductivity (resistivity) present in $\sigma_{\text {set }}$ we compute the numerical error of the real (attenuation ratio) and imaginary (phase difference) part of $q_{Z Z}$ as follows: for every Fourier mode, we replace its numerical contribution into the analytical solution $q_{Z Z}$, obtaining $\widetilde{q_{Z Z}}$. Fig. (4) shows the relative error for the attenuation ratio and phase difference:

$100 \times \frac{\left|\operatorname{Re}\left(\widetilde{q_{Z Z}}\right)-\operatorname{Re}\left(q_{Z Z}\right)\right|}{\left|\operatorname{Re}\left(q_{Z Z}\right)\right|}$, and

$100 \times \frac{\left|\operatorname{Im}\left(\widetilde{q_{Z Z}}\right)-\operatorname{Im}\left(q_{Z Z}\right)\right|}{\left|\operatorname{Im}\left(q_{Z Z}\right)\right|}$,

for two different material resistivities. Finally, we select the maximum element size $h$ such that all errors remain below $1 \% / N_{F}$ for all field components and all resistivities included in our rock formation.

Polynomial order of approximation We allow for arbitrary orders of approximation over each element. Taking advantage of this flexibility, we assign increasingly higher-order polynomial approximations to elements closer to the well trajectory (see Fig. 5). First, we define

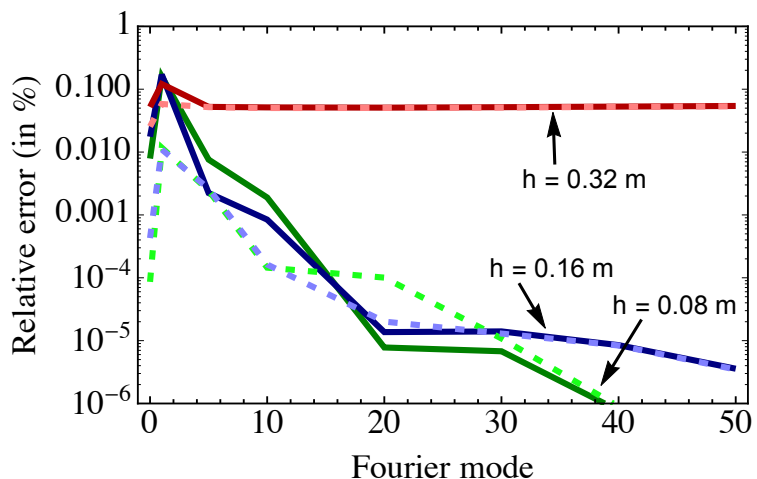

(a) $\rho_{h}=\rho_{v}=100 \Omega \cdot m$.

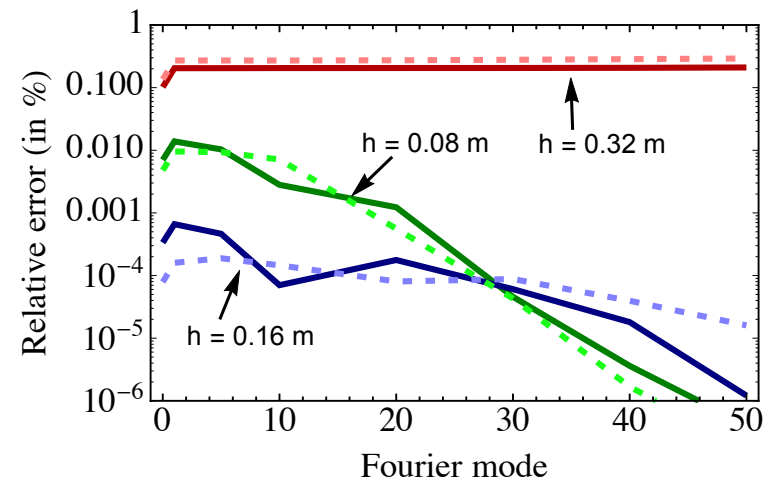

(b) $\rho_{h}=\rho_{v}=0.1 \Omega \cdot m$.

Fig. 4: Relative numerical error (in \%) of the real and imaginary part of $q_{Z Z}$ for different Fourier modes $\beta$. Continuous lines correspond to attenuation ratio (real part) and dotted lines to phase differences (imaginary part).

a distance function dist from the center $\mathbf{x}_{e}$ of a given element elem with respect to the well trajectory $\mathbf{X}_{\text {pos }}$, defined as:

$\operatorname{dist}\left(\mathbf{x}_{\text {elem }}, \mathbf{X}_{\text {pos }}\right)=\min _{\mathbf{x}_{\mathrm{t}}^{(i)} \in \mathbf{X}_{\text {pos }}}\left\|\mathbf{x}_{\text {elem }}-\mathbf{x}_{\mathrm{t}}^{(i)}\right\|_{2}$

Second, we fix a maximum polynomial degree $p_{\max }$ and $p_{\max }$ regions with increasing associated distances $d_{p_{\max }}<d_{p_{\max 1}}<\ldots<d_{p_{2}}<d_{p_{1}}=\infty$. In our case, $p_{\max }=6$. Finally, we define the following piecewise monotonically-decreasing function $p_{\text {order }}$ that assigns a polynomial order to a given element elem as a function of its distance from its central position $\mathbf{x}_{e}$ to the well 


$$
\begin{aligned}
& \text { trajectory } \mathbf{X}_{\text {pos }}: \\
& p_{\text {order }}\left(\mathbf{x}_{\text {elem }}, \mathbf{X}_{\text {pos }} ; p_{\max }\right)= \\
& \begin{cases}p_{\max }, & \text { for } \operatorname{dist}\left(\mathbf{x}_{\text {elem }}, \mathbf{X}_{\text {pos }}\right)<d_{p_{\max }} \\
p_{\max -1}, & \text { for } d_{p_{\max }} \leq \operatorname{dist}\left(\mathbf{x}_{\text {elem }}, \mathbf{X}_{\text {pos }}\right) \leq d_{p_{\text {max }-1}} \\
\cdots & \text { for } d_{p_{2}} \leq \operatorname{dist}\left(\mathbf{x}_{\text {elem }}, \mathbf{X}_{\text {pos }}\right)\end{cases}
\end{aligned}
$$

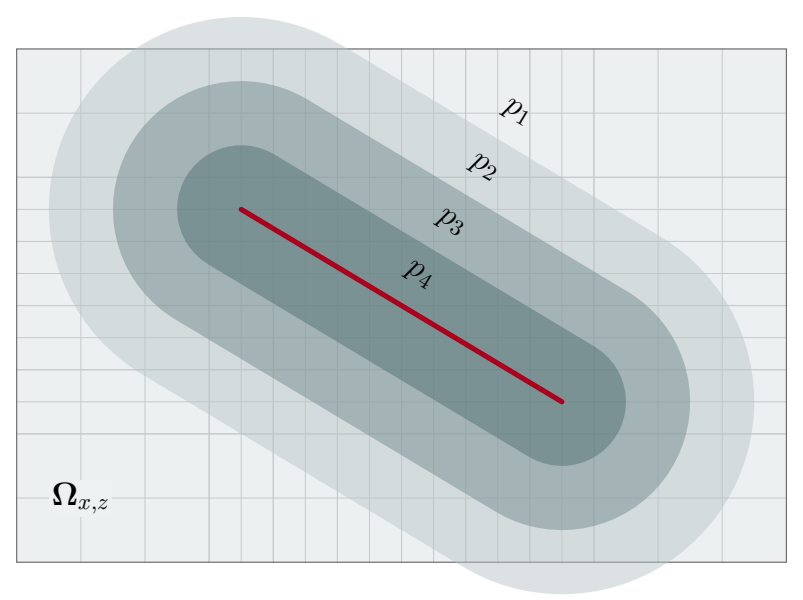

Fig. 5: Assignment of different polynomial orders of approximation to each element according to its distance from the well trajectory (in red).

As a consequence of using a tensor product structure for the grid, unnecessarily small and elongated elements appear in some zones of the domain (e.g., central-top part of Fig. 3). This causes overhead in the simulations. However, the cost of the extra unknowns introduced is alleviated by concentrating most unknowns only in the proximity of the logging instrument via the use of higher polynomial orders of approximation.

Sub-trajectories For a given Fourier mode, Eq. (11) involves the solution of a problem with multiple righthand sides (RHS). Thus, it is possible to perform only once the matrix factorization for all logging positions along the well trajectory. However, this is inconvenient as the computational cost for solving such system grows faster than linearly with the number of unknowns (see [8]). Alternatively, factorizing the matrix for every position is also suboptimal because we would need to perform as many matrix factorizations as number of logging positions.

To minimize the computational cost, we split the logging trajectory into several sub-trajectories. This idea is illustrated in Fig. 6. The optimal number of positions (i.e., the one that minimizes computational time) per sub-trajectory is highly dependent upon dip angle. Experimentally, we have observed that for a vertical well, the optimum number of positions for a single grid is approximately 50, whereas for a 45-degree deviated well (worst case scenario), this number is approximately 10. Selecting 25 positions per grid seems a good practical choice because our numerical estimates indicate that it is within $20 \%$ of the optimum value. In order

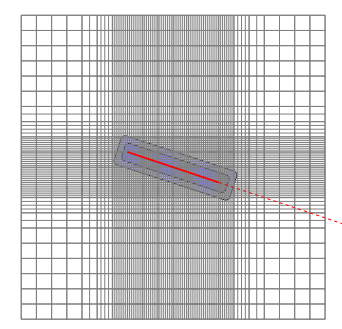

(a) First sub-trajectory.

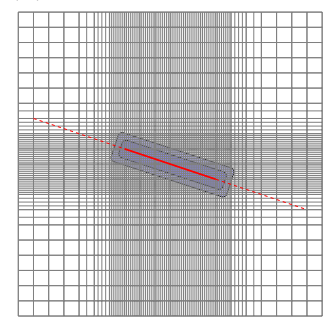

(b) Second sub-trajectory.

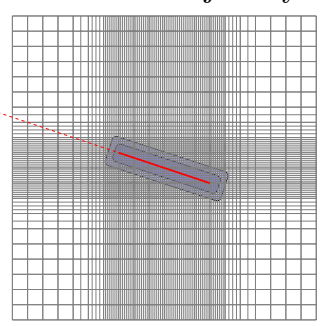

(c) Third sub-trajectory.

Fig. 6: A trajectory that is subdivided into three subtrajectories.

to simulate an apparent resistivity log along the well trajectory, we need to solve

$$
N_{\text {problems }}:=N_{T X} N_{\text {pos }}\left(N_{F}+1\right)
$$

2.5D problems, where $2 N_{F}+1$ denotes the number of modes considered in the truncated Fourier series expansion. For each problem, the number of elements in the $\mathrm{x}$-direction is given by

$$
N_{x} \approx s_{x} / h_{x}+\left(S_{x}-s_{x}\right) / H_{x}
$$

An analogous formula holds for $N_{y}$. If we assume a constant number of degrees of freedom per element along 
each direction, say $\bar{p}$, we estimate that the cost in terms of Floating Point Operations (FLOPs) of a direct solver (see [26]) is given by:

FLOPs $\approx$

$$
\begin{gathered}
C(\underbrace{\mathcal{O}\left(\bar{p}^{6} N_{\text {elements }}+\bar{p}^{3} \min _{x, y}\left\{N_{x}, N_{y}\right\} N_{x} N_{y}\right)}_{\text {LU Factorization }} \\
+\underbrace{N_{\text {pos }} \bar{p}^{4} N_{x} N_{y}}_{\text {Backward Subst. }}) .
\end{gathered}
$$

However, for each pair of Fourier mode and subtrajectory we have a multiple RHS problem on which we perform the factorization only once.

Interpolation of Fourier modes and truncation of the Fourier series As in [30], we select the range of modes $\left[-N_{F}, \ldots, N_{F}\right], N_{F} \in \mathbb{N}^{+}$for truncating the Fourier series expansion of the solution in Eq. (9) according to: (i) the frequency of operation and again, (ii) the worstcase scenario given by the transversely isotropic conductivity tensor $\boldsymbol{\sigma}_{f}$. Additionally, we take advantage of the smooth decay of the Fourier series in our solution as follows: some Fourier modes are selected to be computed by FEM while the remaining ones are simply interpolated in a logarithmic scale. Again, this is done by analyzing the error of the solution at receivers for different material resistivities. For further details regarding design decisions and estimations, see [30].

Parallelization We take advantage of different levels of parallelism. At the highest possible level (coarse grain parallelism), we assign an independent task to each processor, which consists of solving problem (11) for a specific Fourier mode and logging sub-trajectory.

If the number of processors exceeds the number of tasks (i.e., the number of Fourier modes times the number of sub-trajectories), fine-grain parallelism could easily be achieved via domain decomposition techniques $[29,2]$. Such fine grid parallelism has not been considered here.

Finally, we incorporate a dynamic task scheduler following a master-slave model to guarantee an adequate load balance.

\section{Numerical results}

5.1 Model problem with one geological fault

As a first example, we consider the simple model problem illustrated in Fig. (7). The well trajectory with a constant dip angle of 80 degrees crosses a geological fault and two half a meter thick oil-saturated layers.

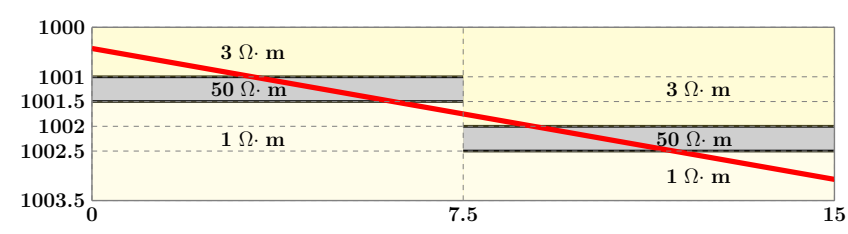

Fig. 7: Model problem with a geological fault: oilsaturated layers are colored in gray.

\subsubsection{Accuracy}

In the following, we consider the response of the LWD instrument described in Section 2 operating at $2 \mathrm{MHz}$. We examine 100 logging positions equally spaced every 0.05 meters along the well trajectory marked in red in Fig. (7). At each position, the logging simulation estimates two different formation (apparent) resistivities associated with the QoI of Eq. (1). The phase difference (imaginary part of (1)) is marked in red in Fig. (8). It provides high vertical resolution. The attenuation (real part of (1)) is marked in blue and provides a greater depth of investigation.

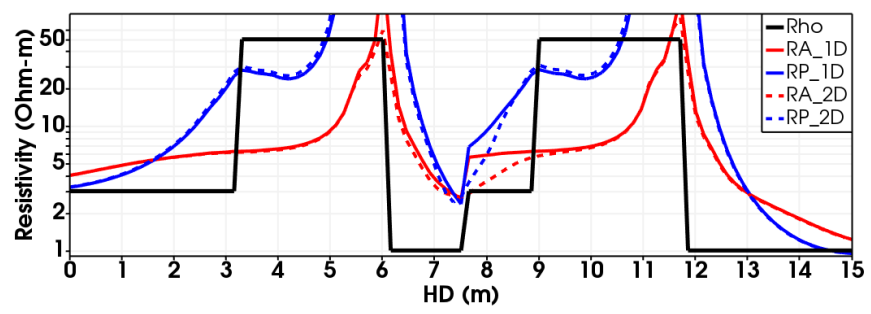

Fig. 8: Attenuation (in red) and phase difference (in blue) apparent resistivity logs simulated for the LWD instrument of Fig. (1) operating at $2 \mathrm{MHz}$. Continuous curves correspond to the $1.5 \mathrm{D}$ method whereas discontinuous curves correspond to the $2.5 \mathrm{D}$ method.

For every measurement, we compare the results obtained with our 2.5D FEM method (2D model) against a semi-analytical $1.5 \mathrm{D}$ code (1D model). The $2.5 \mathrm{D}$ method provides more accurate results than the $1.5 \mathrm{D}$ code nearby the geological fault. 
5.2 Model problem with two geological faults and inclined boundaries

We consider a more challenging model problem illustrated in Fig. (9). This model includes an almost horizontal well, two geological faults and an oil-water contact. The logging trajectory departs from a sandstone layer on the left and penetrates an oil-saturated layer $(\rho=100 \Omega \cdot m)$ three times through the two geological faults, the last one passing through a water-saturated layer $(\rho=0.5 \Omega \cdot m)$. Two inclined boundaries with dip angle of five degrees are also included in the model.

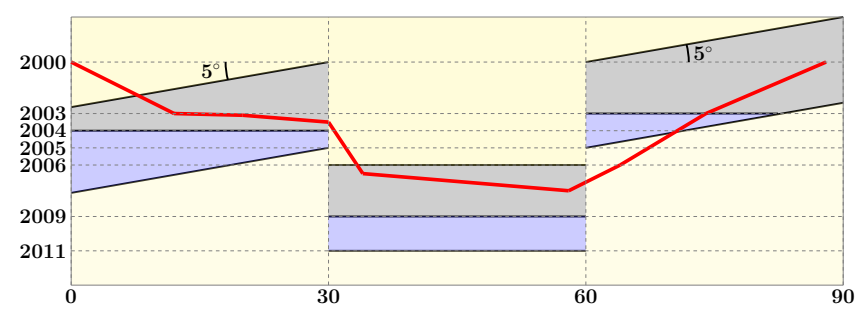

Fig. 9: Model problem with two geological faults and an oil-water contact: oil-saturated layers are colored in gray, water-saturated layers in blue, and sandstone layers in yellow.

\subsubsection{Accuracy and performance}

We consider again the response of the LWD instrument described in Section 2 operating at $2 \mathrm{MHz}$. We examine 2,400 logging positions equally separated every 0.05 meters along the well trajectory marked in red in Fig. (9). As observed in the previous model problem, the phase difference marked in red in Fig. (10) provides high vertical resolution whereas the attenuation marked in blue provides a greater depth of investigation.

We compare the results obtained with our 2.5D FEM method against a semi-analytical 1.5D code and observe two main differences: (i) The 1.5D method produces unphysical spikes as the well trajectory passes through two different layers with inclined planes as expected due to the limitations of 1D models; and (ii) The 2.5D method provides more accurate results than the $1.5 \mathrm{D}$ code nearby the two geological faults.

For this problem, we simulated 11 out of 25 Fourier modes by FEM, saving more than $50 \%$ of computational time. The remaining modes were interpolated in a logarithmic scale (see [30]) using a second-order interpolant. We considered 25 logging positions per subtrajectory. For every mesh, we employed polynomial orders of approximations ranging from $p_{1}$ to $p_{6}$, with

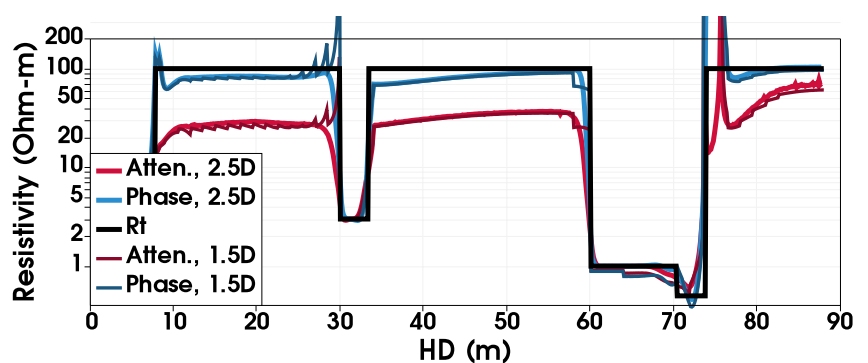

Fig. 10: Attenuation and phase difference apparent resistivity logs simulated for the LWD instrument of Fig. (1) operating at $2 \mathrm{MHz}$. Darker blue and red curves correspond to the 1.5D method whereas lighter blue and red curves correspond to the $2.5 \mathrm{D}$ method.

\begin{tabular}{rrrr}
\hline \#Cores & Time (secs.) & Speedup & Efficiency \\
\hline 1 & 8730 & - & - \\
6 & 1593 & 5,48 & $91 \%$ \\
12 & 875 & 9,98 & $83 \%$ \\
24 & 489 & 17,86 & $74 \%$ \\
48 & 241 & 36,29 & $76 \%$ \\
96 & 118 & 73,97 & $77 \%$ \\
\hline
\end{tabular}

Table 1: Computational time, speedup, and efficiency achieved when using up to 96 cores.

the following distances: $d_{p_{2}}=64 h, d_{p_{3}}=32 h, d_{p_{4}}=$ $16 h, d_{p_{5}}=8 h$, and $d_{p_{6}}=4 h$.

Table 1 shows the computational time and speedup achieved when using different numbers of cores. The cluster employed consists of four computing nodes with 24 cores each (96 in total). Within a single node, the efficiency deteriorates when increasing the number of cores due to the so-called inter-node latency [13]. Once we employ one or more nodes, the efficiency is kept almost constant. This almost perfect scalability is due to the trivial parallelization implemented which requires an almost negligible amount of communication and synchronization among different processors.

Multiple components/frequencies can also be computed in parallel at no extra time provided that an appropriate number of computer cores is employed.

\section{Conclusions}

We developed a method to produce a priori quasi-optimal finite element discretizations for the fast simulation of $2.5 \mathrm{D}$ borehole resistivity measurements. The method is based on the analysis of fundamental solutions of the $2.5 \mathrm{D}$ formulation over multiple homogeneous spaces. 
Regarding the computational mesh, element sizes are selected by means of a non-uniform tensor product structure. An arbitrary polynomial order of approximation can be selected on each element. We proposed a criterion whereby the order of approximation is a function of the distance between the element and the well trajectory.

Self-similar meshes were constructed in terms of Fourier modes, providing a systematic way to truncate the computational domain. Interpolation of Fourier modes decreases the computational time by more than $50 \%$. The trivial parallelization of the library with almost perfect scalability allows us to compute any combinations of triaxial components at no additional time provided that an appropriate number of computing cores is available.

We confirmed the high flexibility of our numerical simulation algorithm for a case with two geological faults, where resistivity logs were obtained at a rate faster than 0.05 seconds per logging position on a modest 96-core machine. The high performance of the library was achieved by enabling a proper adjustment of several parameters and implementing a trivial parallelization.

As future work, this algorithm is intended to be part of a general dimension-adaptive method (DAM) where the dimension of each subproblem represents an input parameter and $1.5 \mathrm{D}, 2.5 \mathrm{D}$, or $3 \mathrm{D}$ simulations are combined to solve general 3D resistivity logging problems over geometrically complex rock formations.

\section{A Fundamental solution of the 2.5D formulation}

To compute the fundamental solution of the $2.5 \mathrm{D}$ formulation, $\mathbf{H}^{f}(\beta)$, we follow the same strategy as in [20] by which the authors computed the fundamental solution for the $3 \mathrm{D}$ transversely isotropic Maxwell's equations. We start from the time-harmonic Maxwell's equations

$$
\nabla \times \mathbf{E}^{f}=-j \omega \mu \mathbf{H}^{f}-j \omega \mu \mathbf{M}^{\mathbf{i m p}},
$$$$
\nabla \times \mathbf{H}^{f}=\left(\boldsymbol{\sigma}_{f}+j \omega \varepsilon\right) \mathbf{E}^{f},
$$

where $\mathbf{E}^{f}$ and $\mathbf{H}^{f}$ are the fundamental solution for the electric and magnetic fields, respectively. Under the assumption that $j \omega \varepsilon$ is much smaller than $\sigma_{h}$ and $\sigma_{v}$, a Hertz vector $\tau=\left[\tau_{x}, \tau_{y}, \tau_{z}\right]^{T}$ and a scalar potential $\phi$ are introduced satisfying

$\boldsymbol{\sigma}_{f} \mathbf{E}^{f}=-j \omega \mu \sigma_{h} \nabla \times \boldsymbol{\tau}$,

and

$\nabla \cdot\left(\boldsymbol{\sigma}_{f} \boldsymbol{\tau}\right)=\sigma_{v} \phi$

Now, the magnetic field solution can be obtained as follows

$\mathbf{H}^{f}=-j \omega \mu \sigma_{h} \boldsymbol{\tau}+\nabla \phi$.
Combining (21) with (17) and using (19) and (20), $\boldsymbol{\tau}$ satisfies the vector equation

$$
\begin{aligned}
& -\boldsymbol{\sigma}_{f}^{-1} \nabla \times\left(\boldsymbol{\sigma}_{f}^{-1} \sigma_{v} \sigma_{h} \nabla \times \boldsymbol{\tau}\right)= \\
& \quad j \omega \mu \sigma_{v} \sigma_{h} \boldsymbol{\sigma}_{f}^{-1} \boldsymbol{\tau}-\boldsymbol{\sigma}_{f}^{-1} \nabla \nabla \cdot\left(\sigma_{f} \boldsymbol{\tau}\right)-\sigma_{v} \boldsymbol{\sigma}_{f}^{-1} \mathbf{M}^{\mathbf{i} \mathbf{m p}} .
\end{aligned}
$$

By taking the Fourier series expansion for $\boldsymbol{\tau}$ and $\mathbf{M}^{\mathbf{i m p}}$ as in Eq. (9), in Eq. (22) we arrive at the following system of equations for each $\tau_{\beta}=\left[\tau_{\beta x}, \tau_{\beta y}, \tau_{\beta z}\right]^{T}$ :

$$
\begin{aligned}
& \frac{\partial^{2} \tau_{\beta x}}{\partial x^{2}}+\frac{\sigma_{v}}{\sigma_{h}} \frac{\partial^{2} \tau_{\beta x}}{\partial z^{2}}-\left(\left(2 \pi \beta / L_{y}\right)^{2}+j \omega \mu \sigma_{v}\right) \tau_{\beta x}= \\
& -\frac{\sigma_{v}}{\sigma_{h}} \frac{1}{L_{y}} \delta\left(x-x_{0}\right) \delta\left(z-z_{0}\right) M_{x} e^{-j 2 \pi \beta y_{0} / L_{y}}, \\
& \frac{\partial^{2} \tau_{\beta y}}{\partial x^{2}}+\frac{\sigma_{v}}{\sigma_{h}} \frac{\partial^{2} \tau_{\beta y}}{\partial z^{2}}-\left(\left(2 \pi \beta / L_{y}\right)^{2}+j \omega \mu \sigma_{v}\right) \tau_{\beta y}= \\
& -\frac{\sigma_{v}}{\sigma_{h}} \frac{1}{L_{y}} \delta\left(x-x_{0}\right) \delta\left(z-z_{0}\right) M_{y} e^{-j 2 \pi \beta y_{0} / L_{y}}, \\
& \frac{\partial^{2} \tau_{\beta z}}{\partial x^{2}}+\quad \frac{\partial^{2} \tau_{\beta z}}{\partial z^{2}}-\left(\left(2 \pi \beta / L_{y}\right)^{2}+j \omega \mu \sigma_{h}\right) \tau_{\beta z}= \\
& -\frac{1}{L_{y}} \delta\left(x-x_{0}\right) \delta\left(z-z_{0}\right) M_{z} e^{-j 2 \pi \beta y_{0} / L_{y}} \\
& +\left(1-\frac{\sigma_{h}}{\sigma_{v}}\right) \frac{\partial}{\partial z}\left(\frac{\partial \tau_{\beta x}}{\partial x}+j 2 \pi \beta / L_{y} \tau_{\beta y}\right) .
\end{aligned}
$$

Each of these three equations with negative real part of the wavenumber corresponds to a modified Helmholtz equation. Now, by solving Eq. (23) and using equations (20) and (21), the fundamental solution for each mode, $\mathbf{H}_{\beta}^{f}$ in terms of any of its nine components of interest, is easy to derive. For example, for $\mathbf{H}_{Z Z}^{f}(\beta)$, we set $M_{x}=M_{y}=0$ and $M_{z} \neq 0$. Then, $\tau_{\beta x}=\tau_{\beta y}=0$ and therefore, proceeding similarly as in [7] for an anisotropic homogeneous Helmholtz equation, it follows that

$$
\tau_{\beta z}=\frac{M_{z}}{2 \pi} \frac{1}{L_{y}} K_{0}\left(\sqrt{-\tilde{k}^{2}} R\right) e^{j 2 \pi \beta y_{0} / L_{y}},
$$

where $K_{0}$ is the modified Bessel function of the second kind,

$$
\begin{gathered}
\tilde{k}^{2}=\tilde{K}\left(-\left(2 \pi \beta / L_{y}\right)^{2}-j \mu \omega \sigma_{h}\right), \\
\tilde{K}=\frac{2}{\lambda_{x}+\lambda_{z} \gamma \gamma^{*}}, \\
R=\sqrt{\left(x+\operatorname{Re}(\gamma) z-x_{0}-\operatorname{Re}(\gamma) z_{0}\right)^{2}+\left(z \operatorname{Im}(\gamma)-z_{0} \operatorname{Im}(\gamma)\right)^{2}},
\end{gathered}
$$

$\gamma$ is the complex root of

$$
\lambda_{x}+\lambda_{z} \gamma^{2}=0
$$

and $\lambda_{x}$ and $\lambda_{z}$ are the coefficients in (23) multyplying the derivatives of second order with respect to $x$ and $z$, respectively. That is, $\lambda_{x}=\lambda_{z}=1$ in the equation for $\tau_{z}$, and $\lambda_{x}=1$ and $\lambda_{z}=\sigma_{v} / \sigma_{h}$ in the first two equations. Using equations (20) and (21), we finally obtain

$$
\mathbf{H}_{Z Z}^{f}(\beta)=-j \omega \mu \sigma_{h} \tau_{\beta z}+\frac{\partial^{2} \tau_{\beta z}}{\partial z^{2}} .
$$

In the above derivation, it is crucial to consider the system of equations in the Fourier domain (23), and find its solutions. Notice that a direct analytical Fourier transform from $\tau$ to $\tau_{\beta z}$ or viceversa is elusive. For validation purposes, we consider two cases: (a) $\sigma_{h}=\sigma_{v}=1 \mathrm{~S} / \mathrm{m}$ and (b) $\sigma_{h}=\sigma_{v}=100 \mathrm{~S} / \mathrm{m}$. Figures (11a) and (11b) compare the analytical vs. the numerical solution for different Fourier modes, showing a perfect agreement in all cases. The derivation for the remaining components is similar. 


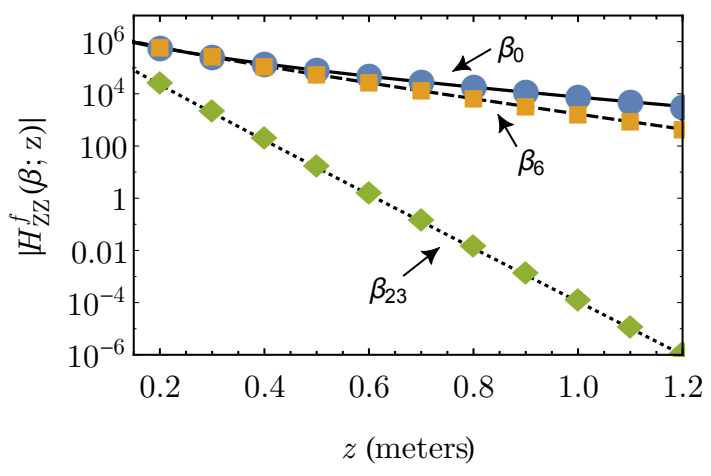

(a) $\rho_{h}=\rho_{v}=1 \Omega \cdot m$.

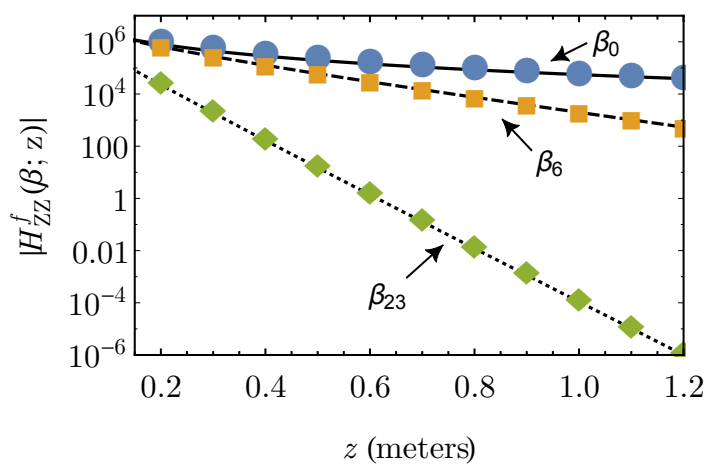

(b) $\rho_{h}=\rho_{v}=100 \Omega \cdot m$.

Fig. 11: Absolute value of $\mathbf{H}_{Z Z}^{f}(\beta)$ for different Fourier modes $\beta$. The marks correspond to numerical values, while the continuous curves correspond to analytical solutions. The solution is displayed over the line $(0,0, z)$, and the source is located at point $(0,0,0.069)$.

Acknowledgements Ángel Rodríguez-Rozas and David Pardo were partially funded by the European Union's Horizon 2020 research and innovation programme under the Marie Sklodowska-Curie grant agreement No 644602, the Projects of the Spanish Ministry of Economy and Competitiveness with reference MTM2016-76329-R (AEI/FEDER, EU) and MTM2016-81697-ERC/AEI, the BCAM Severo Ochoa accreditation of excellence SEV-2013-0323, the Basque Government through the BERC 2014-2017 program, and the Consolidated Research Group Grant IT649-13 on "Mathematical Modeling, Simulation, and Industrial Applications (M2SI). Carlos Torres-Verdín and David Pardo were also funded by the University of Texas at Austin's Research Consortium on Formation Evaluation, jointly sponsored by AkerBP, Anadarko, Aramco, Baker-Hughes, BHP Billiton, BP, China Oilfield Services LTD., Chevron, ConocoPhillips, DEA, ENI, Hess, Inpex, Lundin-Norway, Nexen, Petrobras, Repsol, Shell, Southwestern, Statoil, TOTAL, Wintershall and Woodside Petroleum Limited.

\section{References}

1. Abubakar, A., Habashy, T., Druskin, V., Alumbaugh, D., Zerelli, A., Knizhnerman, L.: Two-and-half-dimensional forward and inverse modeling for marine CSEM problems. In: 76th Ann. International Meeting, Society of Exploration Geophysicists. Expanded Abstracts (2006)

2. Acebrón, J.A., Ángel Rodríguez-Rozas: Highly efficient numerical algorithm based on random trees for accelerating parallel VlasovPoisson simulations. Journal of Computational Physics 250, 224 - 245 (2013)

3. Anderson, B.I.: Modeling and Inversion Methods for the Interpretation of Resistivity Logging Tool Response. DUP Science, Delft, The Netherlands (2001)

4. Bakr, S.A., Pardo, D., Torres-Verdín, C.: Fast inversion of logging-while-drilling resistivity measurements acquired in multiple wells. Geophysics 82(3), E111-E120 (2017)

5. Bittar, M., Aki, A.: Advancement and economic benefit of geosteering and well-placement technology. The Leading Edge 34(5), 524-528 (2015)

6. Calo, V.M., Pardo, D., Paszyński, M.R.: Goal-oriented self-adaptive hp finite element simulation of 3D DC borehole resistivity simulations. Procedia Computer Science 4, 1485 - 1495 (2011). Proceedings of the International Conference on Computational Science, ICCS, 2011

7. Clements, D.L.: Fundamental solutions for second order linear elliptic partial differential equations. Computational Mechanics 22(1), 26-31 (1998)

8. Collier, N.O., Dalcín, L., Pardo, D., Calo, V.M.: The cost of continuity: performance of iterative solvers on isogeometric finite elements. SIAM Journal on Scientific Computing 35(2), A767-A784 (2013)

9. Colton, D.L., Kress, R.: Inverse acoustic and electromagnetic scattering theory. Applied mathematical sciences. Springer-Verlag, New York (1992)

10. Darrigrand, V., Pardo, D., Muga, I.: Goal-oriented adaptivity using unconventional error representations for the 1D Helmholtz equation. Comput. Math. Appl. 69(9), 964-979 (2015)

11. Davydycheva, S.: Two triaxial induction tools: sensitivity to radial invasion profile. Geophysical Prospecting 59(2), 323-340 (2011)

12. Davydycheva, S., Kaminsky, A.: Triaxial induction logging: New interpretation method for biaxial anisotropic formations - part 1. Interpretation 4(2), SF161SF174 (2016). DOI 10.1190/INT-2015-0136.1. URL http://interpretation.geoscienceworld.org/content/4/2/SF161

13. Dror, R., Grossman, J.P., Mackenzie, K., Towles, B., Chow, E., Salmon, J., Young, C., Bank, J., Batson, B., Shaw, D., Kuskin, J.S., Larson, R.H., Moraes, M.A., Shaw, D.E.: Overcoming communication latency barriers in massively parallel scientific computation. IEEE Micro 31(3), 8-19 (2011)

14. Dupuis, C., Denichou, J.M.: Automatic inversion of deepdirectional-resistivity measurements for well placement and reservoir description. The Leading Edge 34(5), 504$512(2015)$

15. Hou, J., Bittar, M.: Correction for the borehole effect of multi-component array induction log data. In: Progress In Electromagnetics Research Symposium Proceedings (2010)

16. Ijasan, O., Torres-Verdín, C., Preeg, W.E.: Inversionbased petrophysical interpretation of logging-whiledrilling nuclear and resistivity measurements. GEOPHYSICS 78(6), D473-D489 (2013)

17. Liu, C.R. (ed.): Theory of Electromagnetic Well Logging. Elsevier (2017)

18. Løseth, L.O., Ursin, B.: Electromagnetic fields in planarly layered anisotropic media. Geophysical Journal International 170(1), 44-80 (2007) 
19. Mallan, R.K., Torres-Verdín, C.: Effects of petrophysical, environmental, and geometrical parameters on multicomponent induction measurements acquired in highangle wells. In: SPWLA 47th Annual Logging Symposium, Society of Petrophysicists and Well-Log Analysts (2006)

20. Moran, J.H., Gianzero, S.: Effects of formation anisotropy on resistivity-logging measurements. Geophysics 44(7), 1266-1286 (1979)

21. Nam, M.J., Pardo, D., Torres-Verdín, C.: Simulation of triaxial induction measurements in dipping, invaded, and anisotropic formations using a fourier series expansion in a nonorthogonal system of coordinates and a selfadaptive hp finite-element method. Geophysics (2010)

22. Nam, M.J., Pardo, D., Torres-Verdín, C.: Simulation of borehole-eccentered tri-axial induction measurements using a fourier hp finite-element method. Geophysics $\mathbf{7 8}(1)$, D41-D52 (2013)

23. Novo, M.S., da Silva, L.C., Teixeira, F.L.: A comparative analysis of krylov solvers for three-dimensional simulations of borehole sensors. IEEE Geoscience and Remote Sensing Letters 8(1), 98-102 (2011)

24. Pardo, D., Calo, V.M., Torres-Verdín, C., Nam, M.J.: Fourier series expansion in a non-orthogonal system of coordinates for simulation of 3D DC borehole resistivity measurements. Computer Methods in Applied Mechanics and Engineering 197, 1906-1925 (2008)

25. Pardo, D., Nam, M.J., Torres-Verdín, C., Hoversten, M.G., Garay, I.: Simulation of marine controlled source electromagnetic measurements using a parallel fourier hp-finite element method. Computational Geosciences 15(1), 53-67 (2011). DOI 10.1007/s10596-010-9195-1. URL https://doi.org/10.1007/s10596-010-9195-1

26. Pardo, D., Paszynski, M., Collier, N., Alvarez, J., Dalcin, L., Calo, V.M.: A survey on direct solvers for galerkin methods. SeMA Journal 57(1), 107134 (2012). DOI 10.1007/BF03322602. URL https://doi.org/10.1007/BF03322602

27. Pardo, D., Torres-Verdín, C.: Fast 1D inversion of logging-while-drilling resistivity measurements for improved estimation of formation resistivity in high-angle and horizontal wells. Geophysics 80(2), E111-E124 (2015)

28. Pardo, D., Torres-Verdín, C., Nam, M., Paszynski, M., Calo, V.: Fourier series expansion in a non-orthogonal system of coordinates for the simulation of 3D alternating current borehole resistivity measurements. Computer Methods in Applied Mechanics and Engineering 197(45), 3836-3849 (2008)

29. Quarteroni, A., Valli, A.: Domain Decomposition Methods for Partial Differential Equations. Oxford Science Publications (1999)

30. Rodríguez-Rozas, Á., Pardo, D.: A priori Fourier analysis for 2.5D finite elements simulations of logging-whiledrilling (LWD) resistivity measurements. Procedia Computer Science 80, $782-791$ (2016)

31. Shen, J., Sun, W.: 2.5-d modeling of cross-hole electromagnetic measurement by finite element method. Petroleum Science 5(2), 126-134 (2008)

32. Steklova, K., Haber, E.: Joint hydrogeophysical inversion: state estimation for seawater intrusion models in 3d. Computational Geosciences 21(1), 7594 (2017). DOI 10.1007/s10596-016-9595-y. URL https://doi.org/10.1007/s10596-016-9595-y

33. Torres-Verdín, C., Habashy, T.M.: Rapid 2.5-dimensional forward modeling and inversion via a new nonlinear scat- tering approximation. Radio Science 29(04), 1051-1079 (1994) 